\title{
Hemozoin "knobs" in Opisthorchis felineus infected liver
}

\author{
Alexandra G. Pershina ${ }^{1,6^{*}}$, Irina V. Saltykova ${ }^{1,7}$, Vladimir V. Ivanov ${ }^{1}$, Ekaterina A. Perina' ${ }^{1}$, Alexander M. Demin², \\ Oleg B. Shevelev³, Irina I. Buzueva ${ }^{4}$, Anton K. Gutakovskii ${ }^{5}$, Sergey V. Vtorushin', Ilya N. Ganebnykh², \\ Victor P. Krasnov ${ }^{2}$, Alexey E. Sazonov ${ }^{1}$ and Ludmila M. Ogorodova ${ }^{1}$
}

\begin{abstract}
Background: Hemozoin is the pigment produced by some blood-feeding parasites. It demonstrates high diagnostic and therapeutic potential. In this work the formation of co-called hemozoin "knobs" - the bile duct ectasia filled up by hemozoin pigment - in Opisthorhis felineus infected hamster liver has been observed.

Methods: The $O$. felineus infected liver was examined by histological analysis and magnetic resonance imaging (MRI). The pigment hemozoin was identified by Fourier transform infrared spectroscopy and high resolution electrospray ionization mass spectrometry analysis. Hemozoin crystals were characterised by high resolution transmission electron microscopy.

Results: Hemozoin crystals produced by O. felineus have average length $403 \mathrm{~nm}$ and the length-to-width ratio equals 2.0. The regurgitation of hemozoin from parasitic fluke during infection leads to formation of bile duct ectasia. The active release of hemozoin from $\mathrm{O}$. felineus during in vitro incubation has also been evidenced. It has been shown that the hemozoin knobs can be detected by magnetic resonance imaging.

Conclusions: In the paper for the first time the characterisation of hemozoin pigment extracted from liver fluke $O$. felineus has been conducted. The role of hemozoin in the modification of immune response by opisthorchiasis is assumed.
\end{abstract}

Keywords: Hemozoin, Trematode, Opisthorchis felineus, Bile duct ectasia, Magnetic resonance imaging (MRI), Transmission electron microscopy (TEM)

\section{Background}

The feature of metabolism of parasites is the potential target for its detection and therapy. Hemozoin, also known as "malaria pigment", is a byproduct, formed from the toxic heme moiety released during the digested hemoglobin detoxification. Therefore hemozoin formation is an adaptation to hematophagy. This pigment first identified and described for Plasmodium falciparum has been found in other Plasmodium species and in the series of blood-living parasite organisms: in the intracellular protozoan Haemoproteus columbae [1], in the trematodes Schistosoma mansoni $[2,3]$ and Echinostoma

\footnotetext{
*Correspondence: allysyz@mail.ru

'Siberian State Medical University, 2, Moskovsky trakt, 634050, Tomsk, Russia ${ }^{6}$ National Research Tomsk Polytechnic University, 30, Lenina Ave, Tomsk, 634050, Russia

Full list of author information is available at the end of the article
}

trivolvis rediae [4]. Moreover, hemozoin was found in insect ectoparasite Rhodnius prolixus [5].

Hemozoin is a unique biocrystal, a distinguishing trait of some blood-feeding organisms [6], it demonstrates high diagnostic and therapeutic potential. Hemozoin has been widely discussed as a candidate of target macromolecule for parasitic disease treatment [7-9]. The immunomodulate effects of hemozoin and especially its role in regulation of parasite-host interaction are also of a great interest $[10,11]$. In general hemozoin is recognized as a key factor in the induction of malaria-associated immunosuppression $[12,13]$.

Nowadays, various approaches has been developed to diagnose parasitic infections (mainly malaria) based on hemozoin detection: light microscopy, polarization microscopy, RAMAN spectroscopy [14], chemo-luminescence method [15], flow cytometry [16] and mass spectrometry 
(MS) analysis [17, 18]. The unique physical properties of hemozoin have also been used as a basis for malaria diagnostic, e.g. photoacoustic detection $[19,20]$. Thus magnetic properties of hemozoin have been widely used for magnetic separation of the malaria-infected cells [21-23]. Several diagnostic methods, such as magneto-optic technology test (MOT-test) [24-26], laser induced photothermal heating of hemozoin [6, 27], nuclear magnetic resonance and magnetic resonance relaxometry [28, 29] have been developed on the basis of magnetic properties of hemozoin crystal.

Opisthorchis felineus is a parasitic trematode widespread in Europe and Russia [30], especially in Western Siberia. This liver fluke settles in humans' bile duct and causes the opisthorchiasis similar to the O. viverrini in South-East Asia. O. felineus infects a large number of people with potential fatal consequences. In some areas of Western Siberia (e.g. Tomsk region) up to $32.8 \%$ of the population is infected [31]. Nevertheless, metabolites of $O$. felineus have been poorly studied.

During our study of liver fluke (O. felineus) infection in hamster model we have observed bile duct ecstasias filled by black-brown pigment. In this paper we provide the evidence that the identified pigment is a hemozoin identical to byproduct of $O$. felineus. Thus, the accumulation of the hemozoin in bile duct of O. felineus infected liver leads to the formation of knobs which can be detected by magnetic resonance imaging (MRI).

\section{Methods}

Calcium chloride, sodium hydrogen carbonate, sodium chloride, hematin, sodium hydroxide, sodium dodecyl sulfate (SDS), phosphate buffer saline (PBS, tablet), Triton $\mathrm{X}-100$, dimethyl sulfoxide (DMSO) were purchased from Sigma-Aldrich. Tris (Amresco), EDTA (AppliChem) were applied. All chemicals were used as received. Milli-Q (Millipore) water was used for all experiments.

\section{Experimental opisthorchiasis model}

Metacercariae of $O$. felineus were obtained from naturally infected fish caught from fresh water reservoirs in endemic areas of Western Siberia (Tomsk), Russia. The muscular tissue and the subcutaneous tissue were digested by pepsin-HCl. Viable metacercariae were collected and identified by microscopy. Hamsters Mesocricetus auratus were purchased from the Department of Breeding and maintenance of small laboratory rodents of the Institute of Bioorganic Chemistry Academicians M.M. Shemyakin and U.A. Ovchinnikov. For conducting the experiment 6- to 8-week-old male hamsters were infected intragastrically with 50 metacercariae per hamster. Hamsters were housed five in each cage under conventional conditions and were fed with a stock diet and water ad libitum. Ethical Approval: All experiments and the maintenance of experimental animals were performed according to the guidelines of local Ethics Committee of the Siberian State Medical University (No. 3808 from 15.09.2014).

The infected animals $(n=4)$ were sacrificed at 5, 24 and 48 weeks postinfection, uninfected animals $(n=4)$ were used as control on each stage. Animals were euthanized by deep anesthesia with carbon dioxide. Four lobes of the liver were examined for the presence of ecstasia with black-brown pigment, followed by the histological analysis.

To characterize the pigment (hemozoin) of the adult worms, we collected samples of liver tissues avoiding liver fluke habitat areas and samples of bile ducts with pigment from six uninfected (control) and six infected hamsters at the 5 week postinfection.

\section{Histological analysis}

The liver tissue or fluke was placed into $10 \%$ buffered formalin, and then embedded in paraffin. Tissue sections were cut into 4-5 $\mu \mathrm{m}$-thick slices and stained with hematoxylin and eosin. Histological analysis was performed with the optical microscope Axiostar plus (Carl Zeiss, Germany).

\section{Hemozoin extraction}

To extract hemozoin from tissues approximately $30 \mathrm{mg}$ of tissue was homogenized with the glass beads Precellys Ceramic Kit (Bertin technologies, Belgium) in five volumes of a solution containing $50 \mathrm{mM}$ tris/ $\mathrm{HCl} \mathrm{pH} \mathrm{8.0,}$ $5 \mathrm{mM} \mathrm{CaCl}_{2}, 50 \mathrm{mM} \mathrm{NaCl}$ and $1 \%$ Triton X-100. To extract hemozoin from a fluke, the same procedure was used for one fluke. The homogenate was supplemented with $1 \%$ Proteinase $\mathrm{K}$, incubated overnight at $37{ }^{\circ} \mathrm{C}$ and centrifuged at 11,000 g for $40 \mathrm{~min}$. The supernatant was discarded, the pellet was washed in $2 \%$ SDS, $10 \mathrm{mM}$ tris, $\mathrm{pH} 7.5$ and then in $100 \mathrm{mM} \mathrm{NaHCO}, \mathrm{pH} 9.0$ and roughly washed in the water with subsequent centrifugation for 10,000 $\mathrm{g}$ for $15 \mathrm{~min}$ [15].

\section{Determination of hemozoin concentration}

To determine hemozoin concentration the sample was diluted in $100 \mathrm{mM} \mathrm{NaOH}, 2$ \% SD, 3 mM EDTA and analyzed by spectrophotometric method. Series of hematin $(10 \mu \mathrm{M}-1.2 \mathrm{nM})$ in $100 \mathrm{mM} \mathrm{NaOH}, 2 \%$ SDS and 3 mM EDTA was used as a standard. The unknown hemozoin concentration was calculated from the calibration curve of the hematin concentration (nM) vs optical density $(\lambda=401 \mathrm{~nm})$ [15]. The amount of hemozoin was expressed as nmol (hematin)/mg tissue or nmol (hematin)/fluke. 


\section{O. felineus in vitro incubation}

Adult (5 weeks postinfection) O. felineus flukes were taken from the bile ducts of hamster and incubated for $2 \mathrm{~h}$ in RPMI 1640 culture medium (PanEco, Russia) at $37{ }^{\circ} \mathrm{C}$ in an atmosphere of $5 \% \mathrm{CO}_{2}$. Adult worms were placed in single wells of 12-well plates (SPL Life Science) containing $3 \mathrm{ml}$ RPMI 1640, supplemented with $1 \%(\mathrm{vol} / \mathrm{vol})$ antibiotics $(50 \mathrm{mkg} / \mathrm{ml}$ streptomycin and $50 \mathrm{U} / \mathrm{ml}$ penicillin; PanEco, Russia) and incubated for $72 \mathrm{~h}$ [32].

\section{Fourier transform infrared spectroscopy (FTIR)}

IR spectra were recorded on a FTIR-spectrometer Spectrum One B with diffusion refraction accessory (DRA) (Perkin Elmer, USA) in the range of 4000$400 \mathrm{~cm}^{-1}$ with 128 scans and at $4 \mathrm{~cm}^{-1}$ resolution.

\section{MS analysis}

The extracted hemozoin or commercial hematin $\left(C_{34}\right.$ $\mathrm{H}_{32} \mathrm{~N}_{4} \mathrm{O}_{5} \mathrm{Fe}$ ) samples were dissolved in $0.04 \mathrm{~mL}$ of DMSO, diluted in $0.9 \mathrm{~mL}$ of methanol. The samples were injected using a syringe pump (model 100, KD Scientific Inc., Holliston, MA, USA) at a constant flow rate of $0.24 \mathrm{ml} / \mathrm{h}$. Positive ion mass spectra from 500 to 3000 $(\mathrm{m} / \mathrm{z})$ were obtained on a maXis impact HD ultra-high resolution quadrupole time-of-flight mass spectrometer (Bruker Daltonik $\mathrm{GmbH}$, Germany) equipped with a standard electrospray ionization (ESI) ion source. The Tuning Mix ES-TOF G 1969-85000 (Agilent Technologies) was used for mass calibration. The spectra were processed by Compass for otofSeries 1.7 (oTof Control 3.4; Bruker Compass Data Analysis 4.2).

\section{Transmission electron microscopy (TEM)}

For TEM analysis the samples of O.felineus extracted hemozoin crystals were placed on carbon-coated $\mathrm{Cu}$ grid. The TEM and HRTEM (high resolution TEM) images of hemozoin crystals were obtained using transmission electron microscope JEM-2200FS (JEOL, Japan) with a Cs-corrector at $200 \mathrm{kV}$.

Biological samples (liver, bile duct or O. felineus fluke) were fixed in $4 \%$ p-formaldehyde in Hanks buffer, $\mathrm{pH}$ 7.4. After fixation samples were post-fixed with osmium tetroxide, dehydrated in alcohols of increasing concentration and propylene oxide, and then embedded in the Epon-Araldite mixture. Ultrathin unstained sections were observed in a Jem-1400 (JEOL, Japan) TEM.

\section{MRI in vitro and ex vivo}

All ${ }^{1} \mathrm{H}$-MRI experiments were performed on horizontal tomographic scanner with a magnetic field intensity of 11.7 T (Bruker, Biospec 117/16 USR, Germany) equipped with a transmitter volume $(500.3 \mathrm{MHz}$, with diameter of 72/89 mm, Bruker, Biospec, Germany) and a receiver surface $(500.3 \mathrm{MHz}$, with the size of $123 \times 64 \times 31 \mathrm{~mm}$, Bruker, Biospec, Germany) ${ }^{1} \mathrm{H}$ coils. T1 and T2 relaxation maps of water hemozoin suspension phantoms were recorded in order to investigate the MRI-contrast properties of hemozoin. For ex vivo MRI the liver was extracted from hamster after gas euthanasia and placed in $50 \mathrm{~cm}^{3}$ polypropylene tube in PBS buffer. T1-weighted high resolution images of hamster liver (slice thickness $1 \mathrm{~mm}$; field of view $4.0 \times 4.0 \mathrm{~cm}$; matrix $512 \times 512$ dots) were recorded by RARE (rapid with relaxation enhancement) with the pulse sequence parameters $\mathrm{TR}=1.5 \mathrm{~s}, \mathrm{TE}=9.2 \mathrm{~ms}$. The signal intensities of each ROI (region-of-interest) in the T1 map were measured, T1 relaxation time data were calculated automatically by Paravision 5.0. [33]. Ethical Approval: The experimental protocol has been approved by the Bioethics Review Committee of the Institute of Cytology and Genetics (No. 24 from 28.10.2014).

\section{Statistical analysis}

Statistical analyses were performed using IBM SPSS Statistics for Windows, Version 21.0 (Armonk, NY: IBM Corp.). All parameters were treated as non-parametric data. The data was expressed as median and interquartile ranges. Independent data groups were compared through Mann-Whitney test. The P value below 0.05 was considered as significant.

\section{Results}

\section{Histological analysis of the infected liver}

The visual analysis of $O$. felineus infected liver showed that dark knobs - the bile duct ectasia filled by blackbrown pigment - can be found starting from 5 week postinfection. These knobs increased in size in the liver of infected animals in time of infection (Fig. 1a-c ). It's important to note that the size and number of the knobs varied within the samples of the experimental group. The morphological analysis showed that similar knobs can be visualised not only on the surface but also inside a liver. In the control group of uninfected hamster the formation of the knobs has not been observed. The histological analysis evidenced no pathological changes in the liver and bile duct of the uninfected hamster (Additional file 1: Figure. S1).

Histological analysis data demonstrates periductal fibrosis with inflammatory cell infiltration occurring around the bile ducts of the $O$. felineus infected liver. At the 48 week of infection the mighty pigment clumps in bile duct and gallbladder wall were observed in the liver of infected hamster (Fig. 1d, e). The great congestion of pigment in gallbladder wall leads to chronic cholecystitis with sharply expressed proliferation of the epithelial cells and to the formation of the gallbladder polyp. It is important to note that polyp stroma has been filled with the pigment (Fig. 1e). The same 

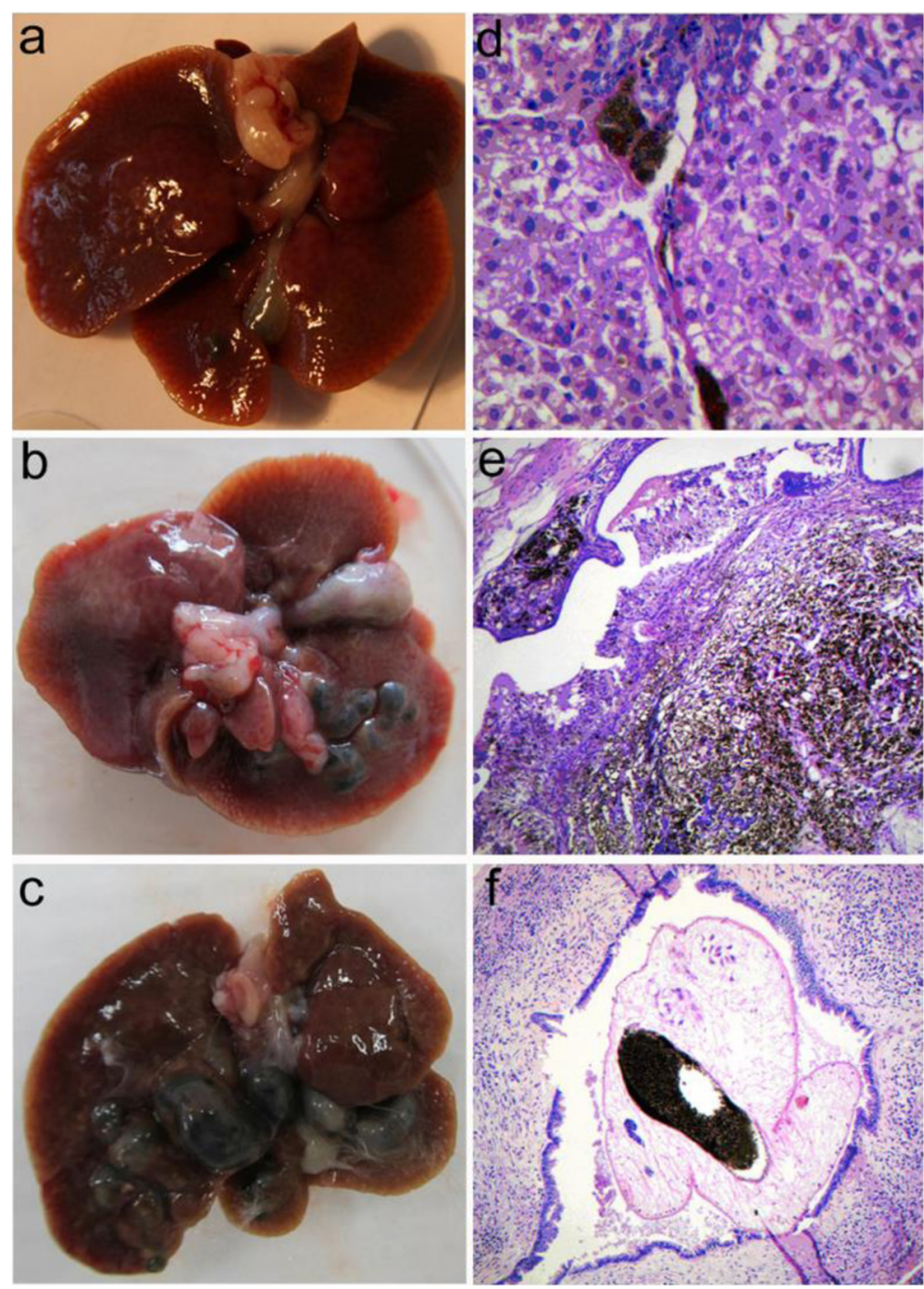

Fig. 1 Histological analysis of the liver of Opisthorchis felineus infected hamster. Macrophotograph of the liver of Opisthorchis felineus infected hamster at (a) 5, (b) 24 and (c) 48 weeks postinfection. The black-brown pigment (hemozoin) forms the increasing hemozoin knobs. Histological analysis evidenced the hemozoin congestions in (d) small bile duct, hematoxylin and eosin staining $(\times 400)$, (e) gallbladder wall and polypoid lesions of the gallbladder, hematoxylin and eosin staining $(\times 200)$ and $(\mathbf{f})$ O. felineus gut, hematoxylin and eosin staining $(\times 400)$

granular black-brown pigment was observed in guts of the fluke (Fig. 1f).

\section{Hemozoin identification}

The bile duct ectasia samples, samples of non-damaged liver tissue and O. felineus flukes were extracted from experimental hamsters and then pigment was extracted according to the protocols for hemozoin purification. The pigment remained insoluble during SDS and sodium bicarbonate at washing steps and it had characteristic hemozoin UV-visible spectra (Additional file 1: Figure. S2). In $0.1 \mathrm{M} \mathrm{NaOH}$ the pigment demonstrated an absorption spectrum identical to monomeric heme [3].
The pigment was also identified by MS and FTIR analysis as hemozoin. For the FTIR analysis the black-brown pigment was extracted from bile duct ectasia. On the FTIR spectra the characteristic hemozoin bands at 1662 and $1209 \mathrm{~cm}^{-1}$ related to $\mathrm{C}=\mathrm{O}$ and $\mathrm{C}-\mathrm{O}$ stretch vibration of the carboxylate group coordinated to the Fe (III) center were detected [34]. The FTIR spectra of hemozoin extracted from bile duct ectasia and $O$. felineus were identical (Fig. 2).

The Laser desorption mass spectrometry (LDMS) method is successfully used for hemozoin detection and identification in biological samples [4, 18, 35]. In these studies the low resolution mass-spectra of hemozoin 


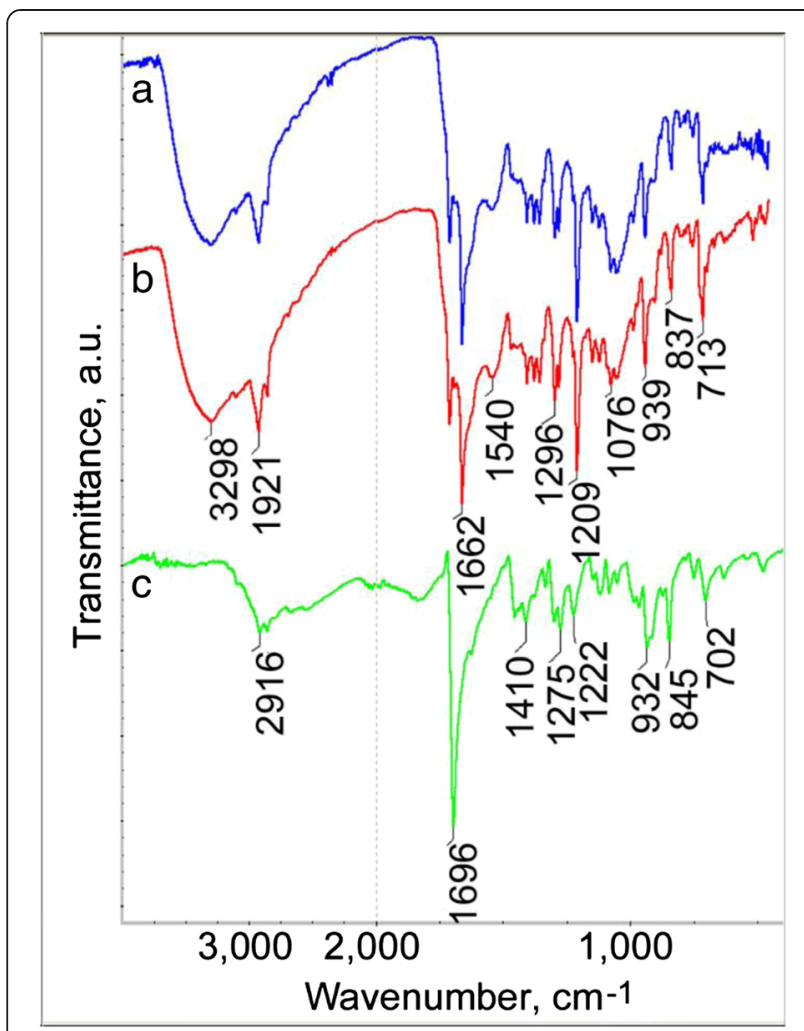

Fig. 2 Fourier transform infrared spectroscopy (FTIR) of hemozoin. FTIR spectra of (a) hemozoin extracted from bile duct ectasia of infected hamster liver, (b) hemozoin extracted from Opisthorchis felineus and (c) commercial hematin (Sigma-Aldrich)

were obtained via direct laser ultraviolet desorption. The mass-spectra exhibited a radical molecular cation $(\mathrm{M}+$ with nominal mass $616 \mathrm{Da})$, corresponding to an individual ionized heme molecule, and several characteristic heme fragment ions.

In our work the positive high resolution MS spectra were obtained using electrospray ionization (ESI) a socalled 'soft ionization' technique. Therefore the extensive fragmentation has not occurred. During the sample preparation the hemozoin was dissolved in DMSO to heme. In the mass-spectra of the sample the intensive molecular cation $\mathrm{M}+$ at $\mathrm{m} / \mathrm{z} 616.1777$ with satisfactory accuracy corresponds to the expected molecular ion $\left[\mathrm{C}_{34} \mathrm{H}_{32} \mathrm{FeN}_{4} \mathrm{O}_{4}\right]^{+}$with calculated mass $616.1768 \mathrm{Da}$ (Fig. 3). The mass-specter of hematin commercial sample $(+616.1780)$ was similar. Also cluster peak with $\left[\mathrm{C}_{34} \mathrm{H}_{32} \mathrm{FeN}_{4} \mathrm{O}_{4}+\mathrm{DMSO}^{+}\right.$ions mass of $+694.191 \mathrm{Da}$ has been detected in both mass-spectra.

\section{Transmission electron microscopy (TEM) characterization of hemozoin}

Hemozoin crystals produced by the $O$. felineus were lath shaped with lengths about 100-600 nm and average length-to-width ratio about 2.0. The corresponding Fast

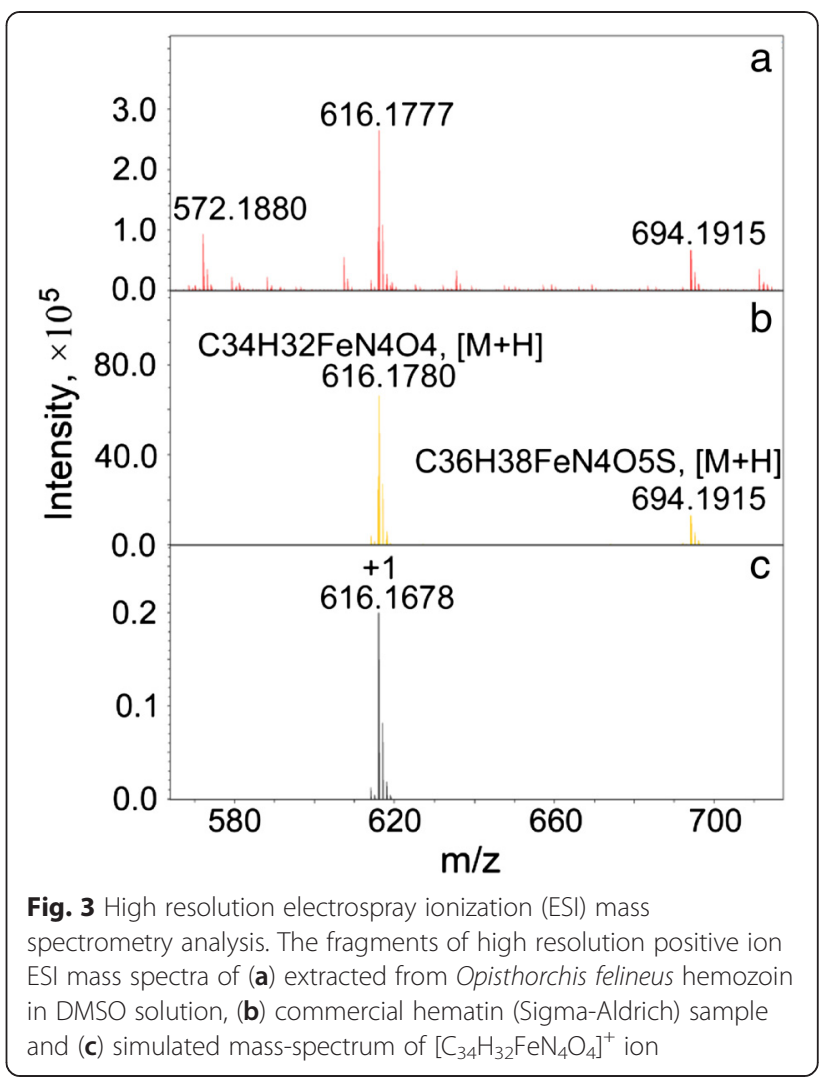

Fourier transform (FFT) image confirmed that the isolated hemozoin crystals exhibited very regular lattice fringes with a spacing of $12.0 \AA$ emphasizing that the crystals tended to lie on their $\{100\}$ or $\{\overline{1} 00\}$ faces (Fig. 4 and Additional file 1: Figure. S3). The determined lattice spacing of hemozoin crystals produced by O. felineus is consistent with the corresponding for synthetic crystals and hemozoin crystals from P. falciparum (varied from 11.9 to $12.6 \AA$ ) [36]. Parameters of external size of extracted hemozoin crystals produced by O. felineus and produced by $P$. falciparum hemozoin are slightly different. The hemozoin crystals produced by $P$. falciparum have average length $500 \mathrm{~nm}$ (from 180 to $1400 \mathrm{~nm}$ ) and length-to-width ratio 3.4. By no surprise since hemozoin crystals extracted from various species such as $P$. falciparum, S. mansoni and $H$. columbae also have different morphology [1].

\section{Hemozoin dynamics in 0 . felineus}

In TEM examination of $O$. felines ultrastructure a large number of hemozoin crystals were found at the bottom of parasite gut and less in the oral sucker area (Fig. 5a). The average length of the hemozoin crystals was around $403(232 \div 615) \mathrm{nm}$, however it varied from about 99-1024 nm (Additional file 1: Figure. S4), an average length-to-width ratio was equal to $2.0(1.6 \div 2.6)$. 


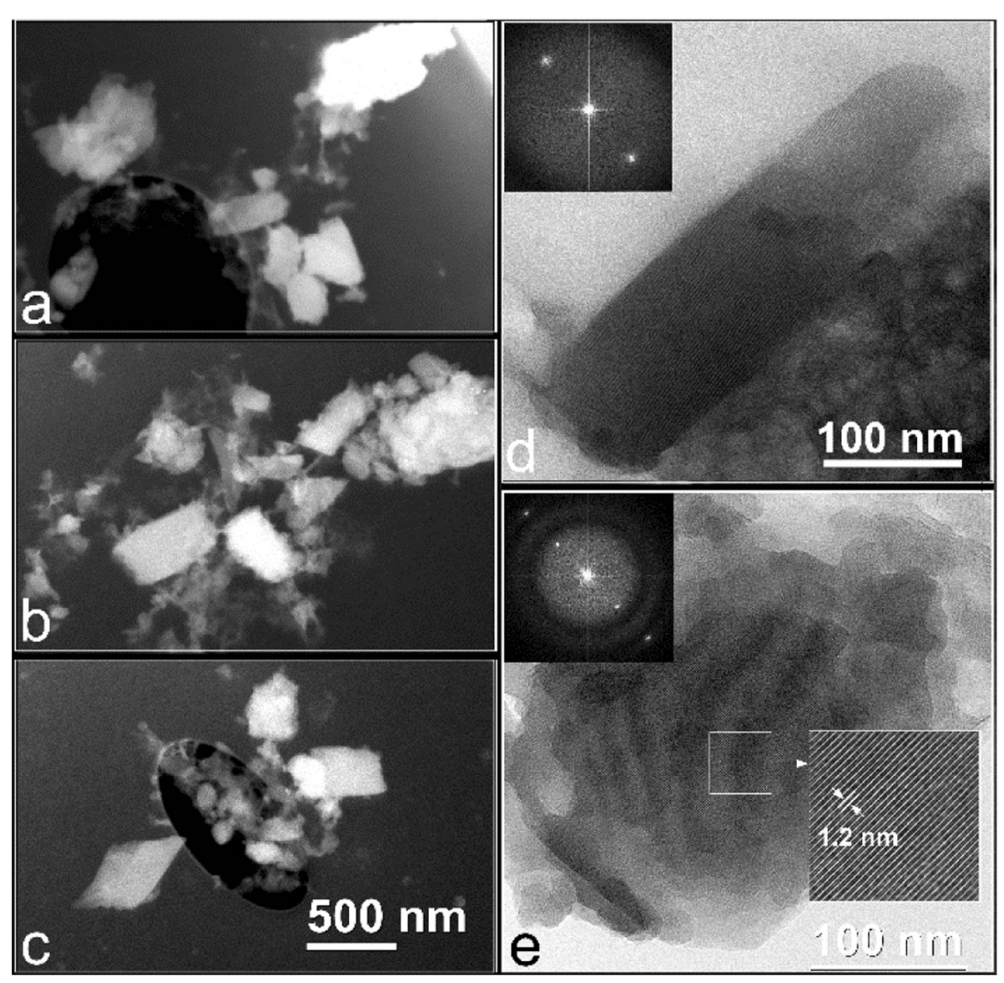

Fig. 4 Transmission electron microscopy (TEM) analysis of extracted hemozoin crystals. (a, b, c) Dark field TEM images of hemozoin crystals extracted from Opisthorchis felineus (d, e) HRTEM image of hemozoin crystals, corresponding FFT image (in the left insert box) and an enlargement of the region (in the right insert box), exhibited regular lattice fringes with a spacing of $12.0 \AA$, confirming that the crystals tended to lie on their $\{100\}$ or $\{100\}$ faces

No crystals were found in utherus, testes, vitelline, nor in a tegument of the fluke. It is important to note that hemozoin crystals were clearly observed in bile duct lumen of the infected animals (Fig. 5b).

The ability of the fluke to regurgitate hemozoin crystals in hosts' bile duct leads to its accumulation in the parasite habitat area and formation of hemozoin knobs. The spectrophotometry revealed that the concentration of hemozoin in the knobs was 73 -fold higher than in the liver parenchyma - $821.1(536.4 \div 934.0)$ and $8.4(7.7 \div 13.2) \mathrm{nmol}$ (hematin)/g, respectively $(p=0.028)$.

Obviously, the concentration of hemozoin in parasite is not constant. Distribution of the hemozoin in adult fluke extracted from the liver of 6 infected hamsters is shown in Fig. 6a. The calculated median of hemozoin concentration in O. felineus was equal to $3.9(2.07 \div 5.09)$ nmol (hematin) per fluke according to spectrophotometry. It was noted that this parameter varied from 0.08 to $7.66 \mathrm{nmol}$ per fluke.

The ability of hemozoin to be released was clearly evident in the cultural experiments. The flukes removed from an infected liver were divided into two groups $(n=$ 11 ); one of them was incubated in RPMI medium for 3 days. After incubation in vitro the hemozoin was practically absent in the preincubated flukes $(p<0.0001)$
(Fig. 6b). Thus the fluke loses the hemozoin during the incubation. This fact is important to consider during any investigation of a parasite or during their extract. It is recommended to exclude the worm preincubation step bearing in mind that hemozoin possess unique properties and contribute to the immune response.

\section{Ex vivo liver MRI}

In MRI phantom experiments of water suspension of the extracted hemozoin we have not observed any contrast properties in both $\mathrm{T} 1$-and T2-weighted sequences. Although the ex vivo MRI of the $O$. felineus infected liver clearly detected significantly decreasing intensity of the signal on T1-weighted image in hemozoin concentration zone (Fig. 7). In hemozoin knobs $\mathrm{T} 1$ values are 5-fold shorter than $\mathrm{T} 1$ of liver parenchyma. These are equal to $279 \mathrm{msec}$ and $1401 \mathrm{msec}$, respectively. Thus, hemozoin knobs can be detected as dark areas in the liver tissue in T1-weighted MRI scans.

We suppose that weaker MRI signal in the zone of hemozoin accumulation in comparison with surrounding tissue is detected due to high hydrophobicity of the pigment. This feature can be used for MRI visualisation of hemozoin clumps for diagnostic of the parasitic diseases. 


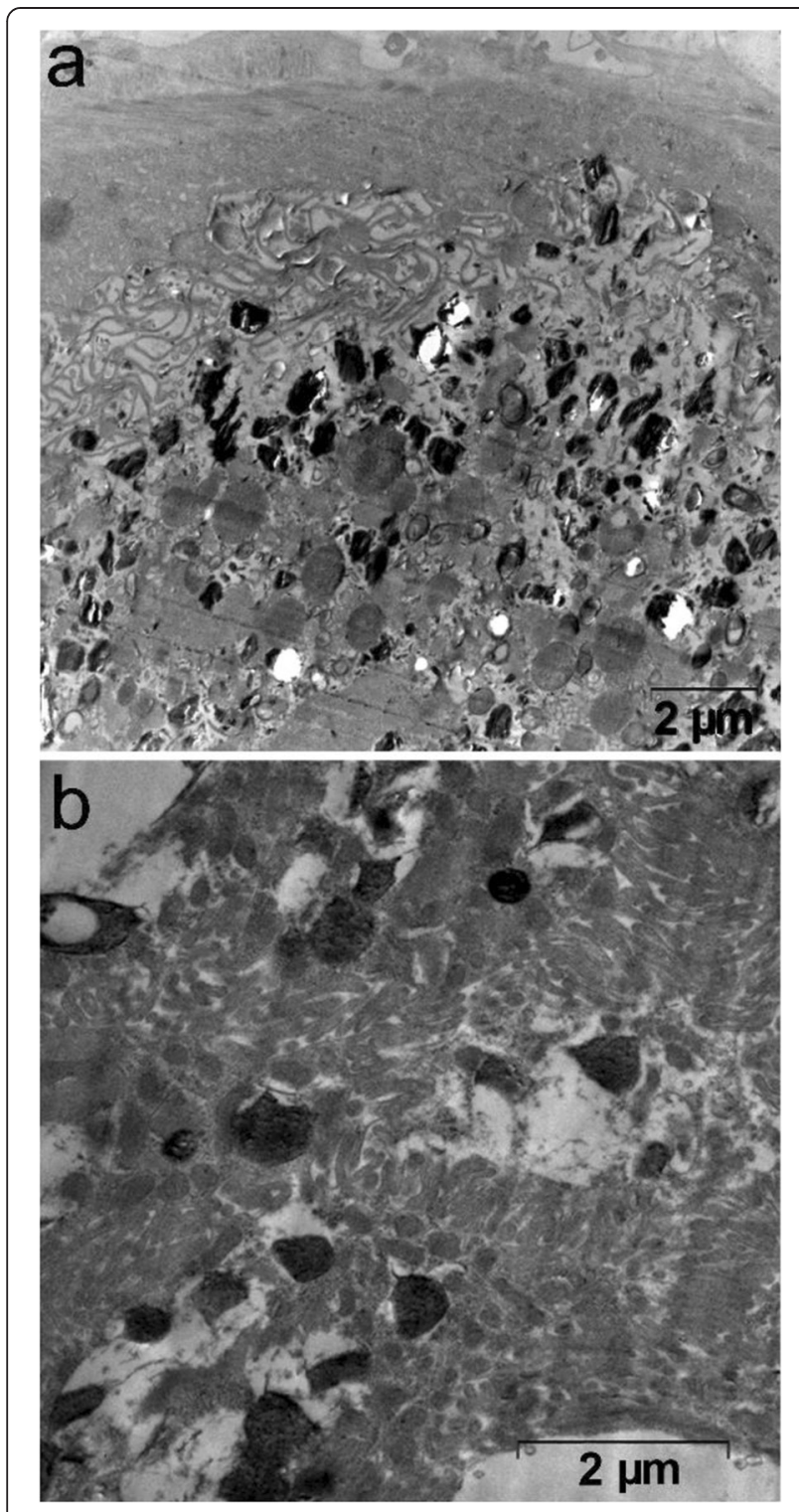

Fig. 5 Transmission electron microscopy (TEM) analysis of hemozoin crystals observed in vivo. TEM images of hemozoin crystals in (a) Opisthorchis felineus gut and (b) bile duct lumen of O. felineus infected hamster (at 5 week postinfection)

\section{Discussion}

O. felineus fluke produces hemozoin similar to other blood-feeding parasites. Previously Sripa and co-workers have observed black-brown pigment in the gut of the adult worm O. felineus. They have assumed that it can be an acid hematin, bilirubin, or even melanin [37].

It is important to emphasize that O. felineus rapidly regurgitated hemozoin crystals. It is well known that blood-living parasite ejects hemozoin in a host bloodstream. In bloodstream hemozoin is captured by macrophages migrating through the body and is accumulated in different organs [15]. The hemozoin is often observed

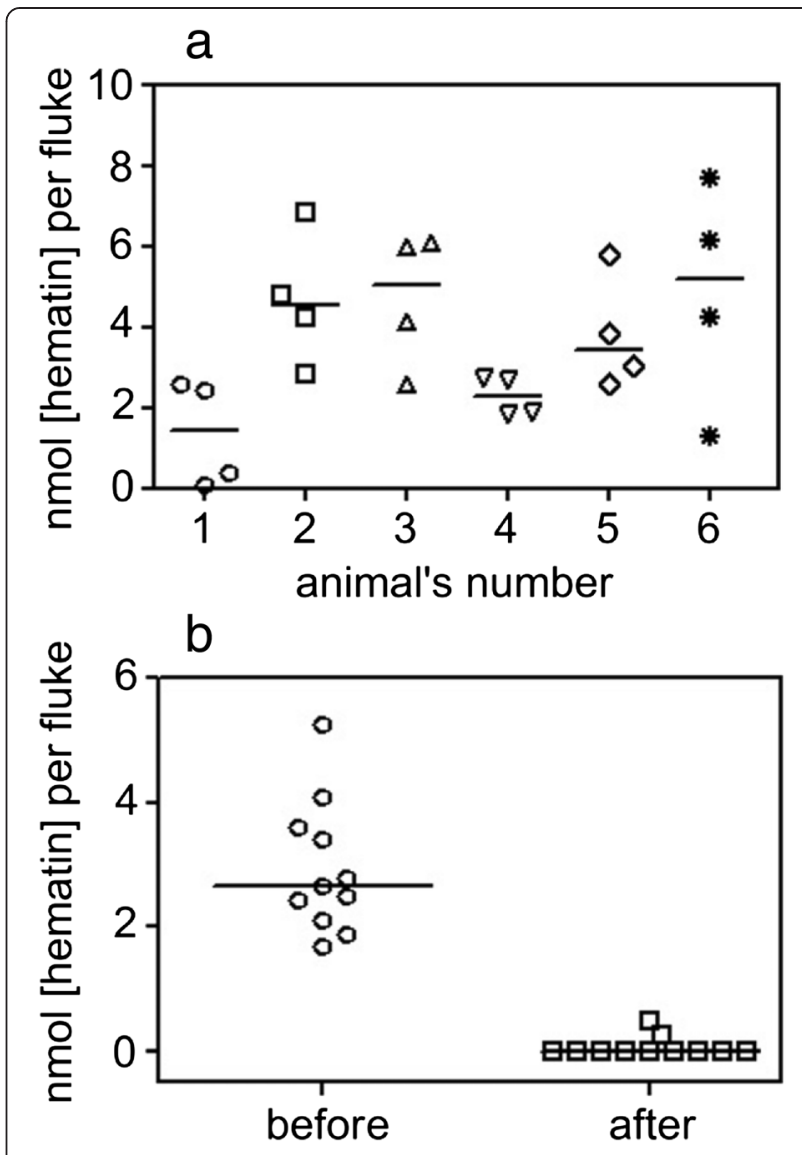

Fig. 6 The hemozoin concentration in Opisthorchis felineus fluke. The hemozoin concentration in Opisthorchis felineus extracted from (a) six infected hamster at 5 week postinfection and (b) an infected hamster liver at 5 week postinfection before and after 3-days in vitro incubation in RPMI $(p<0.0001)$. The Mann-Whitney test was applied

in a liver and a spleen, less in lungs and kidneys during malaria infection [38]. The ability of parasites to regurgitate the hemozoin in blood circulation of host with subsequent accumulation in a liver is described for $S$. mansoni [11]. In the case of opisthorchiasis the hemozoin is accumulated in hosts' bile duct and leads to hemozoin knobs formation. These hemozoin clumps cause the bile duct occlusion, mechanical damage and inflammation (cholecistitis), stimuli proliferation and neoplastic deformation (polyp). The fact of hemozoin detection in gallbladder provides a new insight in the pathogenesis of the gallbladder disease associated with O. felineus infection.

Olivera and co-workers earlier emphasized that hemozoin may play an essential role in the host-protective granulomatous response modulating and moderating [11]. On the other hand, O. felineus is able to modify the host immune response, namely, the opisthorchiasis infection diminishes genetic risk of atopic bronchial asthma [39] and prevents the food sensabilization [40]. Additionally, 

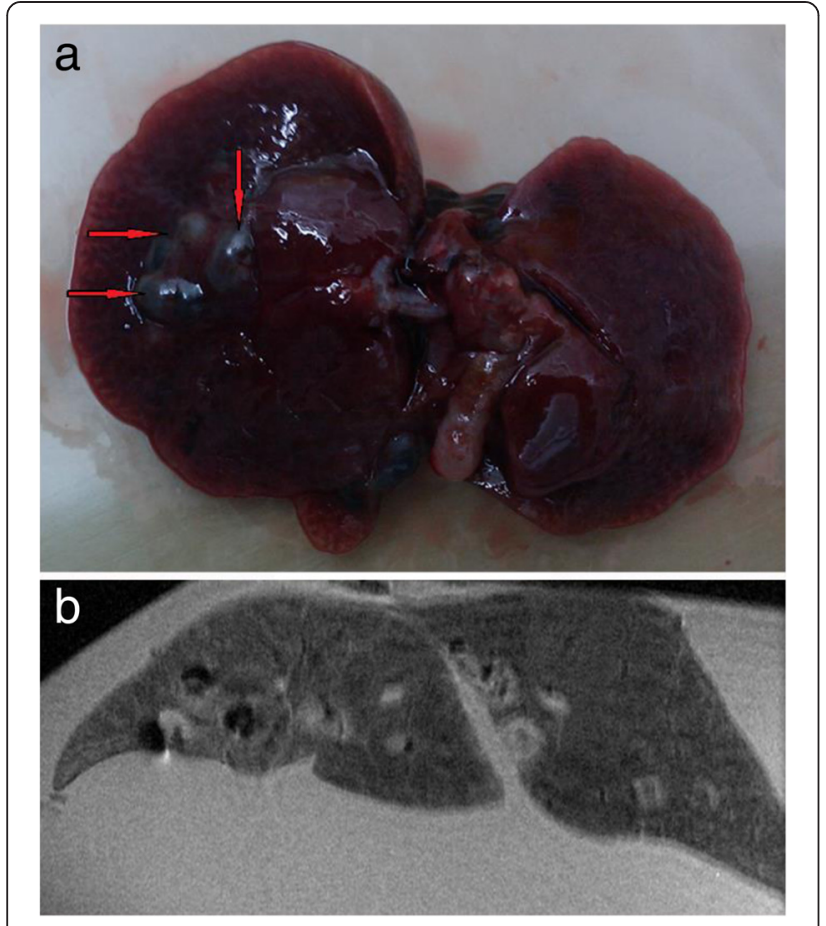

Fig. 7 Detection of hemozoin knobs by magnetic resonance imaging. (a) The macrophotograph of Opisthorchis felineus infected hamster liver at 12 week postinfection. The hemozoin knobs in bile duct are marked by pointer; (b) ex vivo MRI of this liver in PBS buffer. The hemozoin knobs are visualised as dark areas

the monocyte-derived dendritic cells from peripheral blood of bronchial asthma patients stimulated with $O$. felineus extract in vitro exhibit decreased expression level of the most important costimulatory molecules CD86 on the cell surface [41]. Taking into account the immunosuppressive properties of hemozoin [13] and its ability to inhibit human monocytes differentiation and maturation [42], we supposed that the hemozoin production and its excretion can be the drive factor of the immunoregulatory activity of $\mathrm{O}$. felineus. Considering the above, the key question for further research is a way by which the hemozoin crystals could pass a bile duct and be captured by macrophages.

\section{Conclusions}

In summary, we have demonstrated that the liver fluke O. felineus produced a pigment hemozoin specific for some blood-feeding parasites and regurgitated it in a bile duct of host organism. This process leads to formation of massive hemozoin clumps so-called hemozoin knobs. The hemozoin knobs can be detected by T1weigthened MRI. Taken into account the immunosuppressive properties of hemozoin, we can assume that this biomolecule plays a key role in modification of an immune system response during $O$. felineus infection. Thus, the investigation of hemozoin effects on immune system of $O$. felineus infected persons especially in the view of influencing on the progress of comorbidity immunopathology disease is very important.

\section{Additional file}

Additional file 1: Hemozoin "knobs" in Opisthorchis felineus infected liver. Figure S1. Histological analysis of the liver of uninfected hamster (control). Macrophotograph of the liver of uninfected hamster (control) at (a) 5, (b) 24 and (c) 48 weeks after the start of the experiment. Histological analysis of the liver of uninfected hamster (control) at (d) 5, (e) 24 and (f) 48 weeks after the start of the experiment, hematoxylin and eosin staining (×200). Figure S2.

UV-visible spectra of hemozoin (in water) extracted from Opisthorchis felineus (1) and the same amount of hemozoin dissolved in $\mathrm{NaOH}, \mathrm{SDS}$, EDTA buffer. Figure S3. The intensity profile of the designated area in Fig. 2e in article. Figure S4. The histogram of hemozoin crystals length in Opisthorchis felineus gut (PDF 529 kb)

\section{Abbreviations}

SDS: (Sodium dodecyl sulfate); PBS: (Phosphate buffer saline);

DMSO: (Dimethyl sulfoxide); EDTA: (Ethylenediaminetetraacetic acid); FTIR: (Fourier transform infrared spectroscopy); MS: (Mass spectrometry); LDMS: (Laser desorption mass spectrometry); DRA: (Diffusion refraction accessory); TEM: (Transmission electron microscopy); HR: (High resolution); FFT: (Fast Fourier transform); MRI: (Magnetic resonance imaging).

\section{Competing interests}

The authors declare that they have no competing interests.

\section{Authors' contributions}

AGP, IVS and WI conceived and designed the experiments, IVS isolated hemozoin, AGP provided spectrophotometric analysis, WI and EAP conducted animal experiments, AMD carried out FTIR analysis, OBS implemented MRI, IIB provided TEM in vivo, AKG performed HR-TEM of crystals, SW conducted histological analysis, ING carried out HR-ESI-MS, AGP and IVS analysed the data and co-wrote the paper, VPK and AES critically revised the manuscript for important intellectual content, LMO supervised the project. All authors discussed the results and commented on the manuscript. All authors have given approval to the final version of the manuscript.

\section{Additional information}

The macrophotographs and histological analysis of the uninfected hamster liver (control group); the histogram of hemozoin crystals length in $O$. felineus gut; UV-visible spectra of hemozoin extracted from O. felineus; the intensity profile of the designated area in HRTEM (Fig. 2e) of hemozoin.

\section{Acknowledgements}

This work was supported by Russian Scientific Fund (grant no.14-15-00247). HRTEM studies were performed using the equipment of CCU

"Nanostructures" (project ID RFMEFI62114X0004). The MRI study was conducted at the Center for Genetic Resources of Laboratory Animals at the Institute of Cytology and Genetics, Siberian Branch, Russian Academy of Sciences (project ID RFMEFI61914X0005 and project ID RFMEFI61914X0010). We acknowledge support and collaboration of the TOPIC (Tomsk OPIsthorchiasis Consortium), www.topic-global.org.

\section{Author details}

'Siberian State Medical University, 2, Moskovsky trakt, 634050, Tomsk, Russia. ${ }^{2}$ Postovsky Institute of Organic Synthesis, UB RAS, 22, S. Kovalevskoy St, 620137 Yekaterinburg, Russia. ${ }^{3}$ Institute of Cytology and Genetics, SB RAS, 10, Lavrentyev Ave, 630090 Novosibirsk, Russia. ${ }^{4}$ Federal State Budgetary Scientific Institution "Scientific Research Institute of Physiology and Basic Medicine", 4, Timakova St, 630117, Novosibirsk, Russia. ${ }^{5}$ Rzhanov Institute of Semiconductor Physics, SB RAS, 13, Lavrentyev Ave, Novosibirsk, 630090, Russia. ${ }^{6}$ National Research Tomsk Polytechnic University, 30, Lenina Ave, Tomsk, 634050, Russia. ${ }^{7}$ National Research Tomsk State University, 36, Lenina Ave, Tomsk, 634050, Russia. 
Received: 3 August 2015 Accepted: 27 August 2015

\section{Published online: 17 September 2015}

\section{References}

1. Chen MM, Shi LR, Sullivan Jr DJ. Haemoproteus and Schistosoma synthesize heme polymers similar to Plasmodium hemozoin and beta-hematin. Mol Biochem Parasitol. 2001;113:1-8.

2. Kloetzel K, Lewert RM. Pigment formation in Schistosoma mansoni infections in the white mouse. Am J Trop Med Hyg. 1966;15:28-31.

3. Oliveira MF, d'Avila JC, Torres CR, Oliveira PL, Tempone AJ, Rumjanek FD, et al. Haemozoin in Schistosoma mansoni. Mol Biochem Parasitol. 2000;111:217-21.

4. Pisciotta JM, Ponder EL, Fried B, Sullivan Jr DJ. Hemozoin formation in Echinostoma trivolvis rediae. Int J Parasitol. 2005;35:1037-42.

5. Oliveira MF, Silva JR, Dansa-Petretski M, de Souza W, Lins U, Braga CM, et al. Haem detoxification by an insect. Nature. 1999;400:517-8.

6. Coronado LM, Nadovich CT, Spadafora C. Malarial hemozoin: From target to tool. Biochim Biophys Acta. 1840;2014:2032-41.

7. Bandyopadhyay U, Dey S. Antimalarial Drugs and Molecules Inhibiting Hemozoin Formation. In: Becker K, editor. Apicomplexan Parasites: Molecular Approaches toward Targeted Drug Development. Weinheim: Wiley-VCH Verlag GmbH \& Co KGaA; 2011. p. 205-34.

8. Buller R, Peterson ML, Almarsson O, Leiserowitz L. Quinoline Binding Site on Malaria Pigment Crystal: A Rational Pathway for Antimalaria. Drug Design Cryst Growth Des. 2002;2:553-62.

9. Lukianova-Hleb EY, Lapotko DO. Malaria theranostics using hemozoin-generated vapor nanoknobs. Theranostics. 2014;4:761-9.

10. Schwarzer E, Skorokhod OA, Barrera V, Arese P. Hemozoin and the human monocyte-a brief review of their interactions. Parassitologia. 2008;50:143-5.

11. Truscott M, Evans DA, Gunn M, Hoffmann KF. Schistosoma mansoni hemozoin modulates alternative activation of macrophages via specific suppression of retnla expression and secretion. Infect Immun. 2013;81:133-42.

12. Millington $\mathrm{OR}$, Gibson VB, Rush CM, Zinselmeyer BH, Phillips RS, Garside P, et al. Malaria impairs T cell clustering and immune priming despite normal signal 1 from dendritic cells. PLoS Pathog. 2007:3:1380-7.

13. Scorza T, Magez S, Brys L, De Baetselier P. Hemozoin is a key factor in the induction of malaria-associated immunosuppression. Parasite Immunol. 1999;21:545-54.

14. Yuen C, Liu Q. Magnetic field enriched surface enhanced resonance Raman spectroscopy for early malaria diagnosis. J Biomed Opt. 2012;17:017005. doi:10.1117/1.JBO.17.1.017005.

15. Deroost K, Lays N, Noppen S, Martens E, Opdenakker G, Van den Steen PE. Improved methods for haemozoin quantification in tissues yield organ-and parasite-specific information in malaria-infected mice. Malar J. 2012;11:166. http://www.malariajournal.com/content/11/1/166.

16. Grobusch MP, Hänscheid T, Krämer B, Neukammer J, May J, Seybold J, et al. Sensitivity of Hemozoin Detection by Automated FlowCytometry in Non- and Semi-Immune Malaria Patients. Cytometry Part B. 2003;55B:46-51.

17. Demirev PA, Feldman AB, Kongkasuriyachai D, Scholl P, Sullivan Jr DJ, Kumar N. Detection of Malaria Parasites in Blood by Laser Desorption Mass Spectrometry. Anal Chem. 2007;74:3262-6.

18. Demirev PA. Mass spectrometry for malaria diagnosis. Expert Rev Mol Diagn. 2002:4:821-9.

19. Saha RK, Karmakar S, Roy M. Computational investigation on the photoacoustics of malaria infected red blood cells. PLoS One. 2012;7:e51774. doi:10.1371/journal.pone.0051774.

20. Samson EB, Goldschmidt BS, Whiteside PJ, Sudduth AS, Custer JR, Beerntsen B, et al. Photoacoustic spectroscopy of $\beta$-hematin. J Opt. 2012;14:ii 065302. doi:10.1088/2040-8978/14/6/065302.

21. Kim CC, Wilson EB, DeRisi JL. Improved methods for magnetic purification of malaria parasites and haemozoin. Malar J. 2010;9:17. doi:10.1186/1475-2875-9-17

22. Kim J, Massoudi M, Antaki JF, Gandini A. Removal of malaria-infected red blood cells using magnetic cell separators: a computational study. Appl Math Comput. 2012;218:6841-50.

23. Paul F, Roath S, Melville D, Warhurst DC, Osisanya JO. Separation of malaria infected erythrocytes from whole blood: use of a selective high-gradient magnetic separation technique. Lancet. 1981;2:70-1.

24. Newman DM, Matelon RJ, Wears ML, Savage LB. The in vivo diagnosis of malaria: feasibility study into a magneto-optic fingertip probe. IEEE J Sel Top Quantum Electron. 2010;16:573-80.
25. Newman DM, Heptinstall J, Matelon RJ, Savage L, Wears ML, Beddow J, et al. A magneto-optic route toward the in vivo diagnosis of malaria: preliminary results and preclinical trial data. Biophys J. 2008;95:994-1000.

26. Butykai A, Orbán A, Kocsis V, Szaller D, Bordács S, Tátrai-Szekeres E, et al. Malaria pigment crystals as magnetic micro-rotors: key for high-sensitivity diagnosis. Scientific Reports. 2013;3:1431. doi:10.1038/srep01431.

27. Lukianova-Hleb EY, Campbell KM, Constantinou PE, Braama J, Olson JS, Ware RE, et al. Hemozoin-generated vapor nanoknobs for transdermal reagent- and needle-free detection of malaria. PNAS. 2014;11:900-5.

28. Karl S, Gutiérrez L, House MJ, Davis TM, St Pierre TG. Nuclear Magnetic Resonance: A Tool for Malaria Diagnosis? Am J Trop Med Hyg. 2011;85:815-7.

29. Peng WK, Kong TF, Ng CS, Chen L, Huang Y, Bhagat AA, et al. Micromagnetic resonance relaxometry for rapid label-free malaria diagnosis. Nat Med. 2014;9:1069-73.

30. Ogorodova LM, Fedorova OS, Sripa B, Mordvinov VA, Katokhin AV, Keiser J, et al. Opisthorchiasis: An Overlooked Danger. PLoS Negl Trop Dis. 2015;9(4):e0003563. doi:10.1371/journal.pntd.0003563.

31. Ogorodova LM, Freidin MB, Sazonov AE, Fedorova OS, Gerbek IE, Cherevko NA, et al. A pilot screening of prevalence of atopic states and opisthorchosis and their relationship in people of Tomsk Oblast. Parasitol Res. 2007;101:1165-8.

32. Duthaler U, Smit TA, Keiser J. In Vivo and In Vitro Sensitivity of Fasciola hepatica to Triclabendazole Combined with Artesunate, Artemether, or OZ78 Antimicrob Agents. Chemother. 2010;54:4596-604.

33. Toth E, Helm L, Merbach AE. Relaxivity of MRI contrast agents. Top Curr Chem. 2002;221:61-101.

34. Slater A, Swiggard WJ, Orton BR, Flitter WD, Goldberg DE, Cerami A, et al. An iron-carboxylate bond links the heme units of malaria pigment. Proc Natl Acad Sci U S A. 1991;88:325-9.

35. Scholl PF, Kongkasuriyachai D, Demirev PA, Feldman AB, Lin JS, Sullivan Jr $D J$, et al. Rapid detection of malaria infection in vivo by laser desorption mass spectrometry. Am J Trop Med Hyg. 2004;71:546-51.

36. Ambele MA, Sewell BT, Cummings FR, Smith PJ, Egan TJ. Synthetic Hemozoin ( $\beta$-Hematin) Crystals Nucleate at the Surface of Neutral Lipid Droplets that Control Their Sizes. Cryst Growth Des. 2013;13:4442-52.

37. Lvova MN, Tangkawattana S, Balthaisong S, Katokhin AV, Mordvinov VA, Sripa B. Comparative histopathology of Opisthorchis felineus and Opisthorchis viverrini in a hamster model: An implication of high pathogenicity of the European liver fluke. Parasitol Int. 2012;61:167-72.

38. Levesque MA, Sullivan AD, Meshnick SR. Splenic and Hepatic Hemozoin in Mice after Malaria Parasite Clearance. J Parasitol. 1999;85:570-3.

39. Saltykova IV, Ogorodova LM, Bragina EY, Puzyrev VP, Freidin MB. Opisthorchis felineus liver fluke invasion is an environmental factor modifying genetic risk of atopic bronchial asthma. Acta Trop. 2014;139:53-6.

40. Ogorodoava LM, Fedorova OS, Fedotova MM, Evdokimova TA. [Food sensitization in children with Opisthorchis felineus invasion]. Vestn Ross Akad Med Nauk. 2014;1-2:64-8 \{Article in Russian.\}. http://www.spr-journal.ru/ webasyst/pdf/article/2014/NRAMN/VR_12/11.Ogorodova.pdf.

41. Kremer EE, Ogorodova LM, Kirillova NA, Khvorilova KV, Perevozchikova TV, Fait EA. Immunophenotypic characteristic of dendritic cells in bronchial asthma in conditions of extract Opisthorchis felineus in vitro. Vestn Ross Akad Med Nauk. 2013;5:66-70. Article in Russian.

42. Skorokhod OA, Alessio M, Mordmüller B, Arese P, Schwarzer E. Hemozoin (malarial pigment) inhibits differentiation and maturation of human monocyte-derived dendritic cells: a peroxisome proliferator-activated receptor-gamma-mediated effect. J Immunol. 2004;173:4066-74. 